\title{
Crystal structure of carbohydrate esterase SmAcE1 from Sinorhizobium meliloti
}

\author{
Changsuk $\mathrm{Oh}^{1,{ }^{*}}$, Truc Kim${ }^{1}$, T. Doohun $\mathrm{Kim}^{2,}$, Kyeong Kyu Kim ${ }^{1, \S}$ \\ ${ }^{1}$ Department of Molecular Cell Biology, Sungkyunkwan University School of Medicine, Suwon, 16419, Republic of Korea \\ ${ }^{2}$ Department of Chemistry, College of Natural Science, Sookmyung Women's University, Seoul, 04310, Republic of Korea \\ *csoh@skku.edu
}

\begin{abstract}
Green chemistry paradigm has been raised to reduce environmental damages during process of products. American Chemical Society suggested 12 principals such as atom economy, safter chemicals, energy efficiency, degradable products and less hazardous chemical syntheses. One of alternatives for green chemistry is using biocatalysts, whose endogenous characters are attractive in the following aspects: decrease synthesis procedures, less side products, and mild reaction condition. Microbial enzymes are one of promising sources for biocatalysts in industrial processes to produce biofuel from biomass and building blocks. We identified the structure of the carbohydrate esterase, SmAcE1 from Sinorhizobium meliloti. The crystal structure of SmAcE1 was determined at $2.05 \AA$ resolution, and revealed that it belonged to an $\alpha / \beta$ hydrolase fold in GDSL superfamily. It formed a hexameric structure by dimer of trimers with supporting of size exclusion chromatography analysis. Catalytic triad (Ser15, His195 and Asp192) and an oxyanion hole-forming SGNH (Ser15, Gly57, Asn97 and His195) were also conserved in its three dimensional structure. The docking analysis to acetylate substrates showed the hydrophilic residues on its surface are important in substrate binding. The models from crystal structure and docking analysis suggest the industrially applicable potency of SmAcE1 after enhancement of its selectivity and activity by further structure-based engineering.
\end{abstract}

Keywords: Sinorhizobium meliloti, carbohydrate esterase, crystal, docking 